\title{
Application of IoT in the COVID-19 Pandemic
}

\author{
B. Shoban Babu and Prince Patel
}

\begin{abstract}
As we all know, the Internet has altered everything, and the Internet of Things has given us hope for a bright future of the Internet with Machine-toMachine (M2M) connectivity. This review study demonstrated that smart systems based on the Internet of Things are feasible and economical to build (IoT). In the field of healthcare, the Internet of Things has made significant progress. This article examines how the Internet of Things (IoT) is revolutionising the healthcare industry by giving huge healthcare advantages to humanity through accessible and practical healthcare solutions specially during the hard coronavirus situation around the world. The purpose of this study is to address the function of IoT in smart hospitals, as well as its importance in dealing with pandemics. Various smart gadgets that can provide a variety of features, such as adequate monitoring of high-risk patients, tracking their bio-metric measurements, and gathering real-time data, can be used to serve community-specific demands during pandemic spread. We've also looked into other plans that can detect unforeseen events utilising a variety of sensors and display the information gathered on an LED display. The results of observational studies have indicated a high level of agreement with the hypothetical claims.
\end{abstract}

Key words - Coronavirus, Pandemic, Internet of Things.

\section{INTRODUCTION}

Smart technology's applications in the sphere of healthcare are spreading at an ever-increasing rate, thanks to the rise of smart technologies. The phrase "smart hospital" refers to a smart healthcare infrastructure that connects all healthcare stakeholders via Internet of Things (IoT) devices [1]. With this collaboration, the quality and consistency of health services can be enhanced, resulting in better patient care. IoT can be useful in emergency scenarios as well, thanks to wearable gadgets, sensors, smart computing, and remote monitoring capabilities. In the event of a pandemic, IoT-based devices can potentially be used to supply healthcare facilities. Due to a lack of clinical infrastructure, dealing with health crises can be a source of concern for many countries. Clinics and hospitals in many countries become overburdened when the number of patients increases during a pandemic, resulting in a scarcity of resources. Effective vaccine deployment systems, capable of regulating and distributing pandemic vaccines during an

Submitted on August 14, 2021.

Published on September 04, 2021.

B. Shoban Babu

Prince Patel, Charotar

(corresponding e-mail: princepate1221375@gmail.com) emergency, are necessary to address the challenge. Healthcare enterprises are becoming increasingly crucial in a country's overall development, thanks to rapid advancements in technology such as the Internet of Things. Due to a lack of clinical infrastructure, dealing with health crises can be a source of concern for many countries. Clinics and hospitals in many countries become overburdened when the number of patients increases during a pandemic, resulting in a scarcity of resources. Effective vaccine deployment systems, capable of regulating and distributing pandemic vaccines during an emergency, are necessary to address the challenge. Health-care enterprises are becoming increasingly crucial in a country's overall development, thanks to rapid advancements in technology such as the Internet of Things.

\section{INTERNET OF THINGS}

The internet of things (IoT) is a network of networked computers, mechanical and digital machinery, objects, animals, and people with unique identities (UIDs) and the ability to transfer data without the need for human or computer interaction [2] It is possible to make anything, from a medication to a jet, a part of the Internet of Things. An IoT ecosystem is made up of web-enabled smart devices that acquire, send, and act on data from their surroundings using embedded systems such as CPUs, sensors, and communication hardware. By connecting to an IoT gateway or other edge device, IoT devices can share sensor data that is either routed to the cloud for analysis or examined locally. These devices may communicate with one another from time to time and act on the information they receive Although people can interact with the devices to set them up, give them instructions, or retrieve data, the devices do the majority of the work themselves.

In healthcare, IoT offers a number of advantages, including the capacity to more closely monitor patients through data analysis [3]. IoT technologies are frequently used in hospitals to fulfil activities such as pharmaceutical and medical instrument inventory management [4]. Sensors that detect how many people are in a room, for example, can help smart buildings save money on energy. IoT sensors and deployments, such as smart lighting and smart metres, can aid in traffic relief, energy conservation, environmental monitoring and mitigation, and cleanliness in a smart city [5].

To manage the rush of devices, the Internet of Things necessitates massive network scalability. To link devices to IP networks, the IETF 6LoWPAN standard would be utilised [6]. With billions of new devices joining the Internet, IPv6 will play a critical role in network layer scalability. Constrained Application Protocol, ZeroMQ, and 
MQTT, all developed by the Internet Engineering Task Force, would allow for lightweight data delivery.

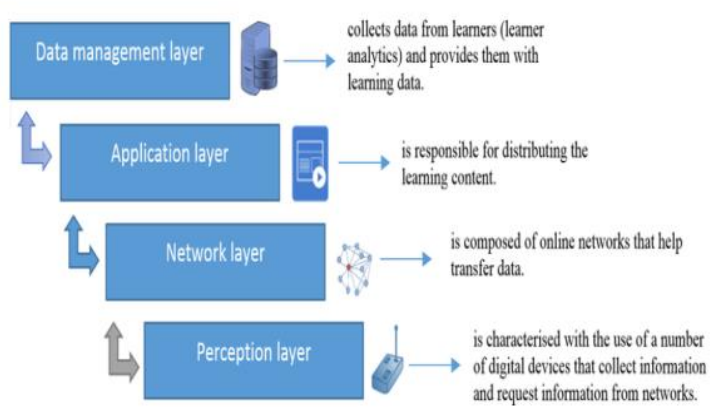

Fig. 1. Layers of IoT Architecture.

In order to provide clinical care to patients, the technological IoT framework is examined. Physiological characteristics can be gathered using a variety of biomedical sensors. Gateways and the cloud are capable of analysing and storing this data, as well as providing it to caregivers for study and evaluation. Even if the situation is lifethreatening.

TABLE I: DIFFERENT COMPONENT USED FOR IOT ARCHITECTURE

\section{Name}

Peripheral

Interface

Controller (PIC)

Radio Frequency

Identification

(RFID)

Wireless Body

Area Network

Wireless Sensor

Network (WSN)

GSM Module

LDR Sensor

Ultrasonic Sensor
IR Sensor IR detectors are small microchips containing a

\section{Discription}

A peripheral interface controller (PIC) is a sort of microcontroller component used in the creation of electronics, computers, robots, and other related technologies. Microchip Technology designed the PIC, which is based on the Harvard Computing architecture, which places code and data in distinct registers to maximise I/O throughput.

RFID stands for Radio Frequency Identification and refers to a wireless technology that consists of two parts: tags and readers. The reader is a device that emits radio waves and receives signals from the RFID tag via one or more antennas. Tags can be passive or active, communicating their identity and other information to nearby readers through radio waves.

A Wireless Body Area Network (WBAN) is a network of independent nodes (such as sensors and actuators) embedded in a person's clothing, on their body, or beneath their skin. The network usually covers the entire human body, with nodes connected by a wireless communication channel.

WSNs are a wireless network of interconnected sensor nodes that collect data from the environment. Lowpower nodes are frequently used, and they are dispersed in an ad hoc, decentralised manner.

A GSM modem, also known as a GSM module, is a physical device that connects to a remote network using GSM mobile phone technology. They are substantially identical to a typical mobile phone in the eyes of the mobile phone network, including the necessity for a SIM to identify themselves to the network.

A photoresistor or a cadmium sulphide $(\mathrm{CdS})$ cell is another name for a Light Dependent Resistor (LDR). A photoconductor is another name for it. It's essentially a photocell that works on the photoconductivity concept. The passive component is essentially a resistor whose resistance lowers as the light intensity falls. photocell that can detect infrared light. They're nearly always employed for remote control detection; every TV and DVD player has one in the front to listen for the clicker's IR signal.

An ultrasonic sensor is a device that uses ultrasonic sound waves to determine the distance to an item. using ultrasonic sound waves.

\section{IOT \& COVID-19}

COVID-19 is a global danger that has affected every country. This section looks at IoT applications that can be used as a road map for dealing with the pandemic by giving a practical plan.

A robotic assistant car controlled by a voice command and powered by a Raspberry Pi can help older persons and the physically impaired. A robotic arm build around IoT infrastructure can connection between a phone and raspberry pi can be controlled through a voice command feature [7], [8]. An IoT-based trash checking system utilizing Arduino or Raspberry pi board and an open IoT stage can be used in hospitals to take out trash [9]. Remote health monitering systems are being developed to keep an eye on patients while mainting appropriate distance [10]. The majority of the time, senior adults and physically challenged persons will require assistance, even for simple things such as getting water, food, and clothing. Jichao Leng et al. [12] developed an IoT system for use in hospitals that has a wide range of applications, including various data collection methods, data uploading to cloud platforms, secure networking, and real-time feedback. The pattern of infectious diseases is predicted to vary, according to Fong, Ignatius $\mathrm{W}$ et al., and this could be a time-consuming process to assess and manage [13]. According to Oshitani, Hitoshi et al. [14], the biggest challenge facing the healthcare industry is the production of high-quality vaccines that are adequate and available in a timely manner, enabling for fast immunisation of entire communities around the world. Another challenge that occurs during pandemics is the timely delivery of pharmaceutical commodities throughout the country, which may need a careful balance of non-pharmaceutical and pharmaceutical activity.

\section{IOT \& SMART HOSPITAL}

Healthcare organisations require appropriate technologies that can be advantageous for tracking and managing the spread of various diseases in order to avoid pandemics and receive real-time recommendations for decision-making. Many governments, however, are having difficulty dealing with pandemics.

Healthcare organisations require appropriate technologies that can be advantageous for tracking and managing the spread of various diseases in order to avoid pandemics and receive real-time recommendations for decision-making. Many governments, on the other hand, are struggling to deal with pandemics. B. G. Ahn, Y. H. Noh et.al. [15] proposed a system for detecting ECG signals in sitting positions that uses a smart chair that detects signals and can be analysed using a monitoring system. In the case of sick people admitted to smart ICUs, I. Chiuchisan, H. N. Costin, et al. developed a framework to avoid any risk. This technology notifies families and doctors of any changes in their health circumstances by tracking bodily gestures [16]. M. S. D. Gupta, V. Patchava, and colleagues [17] created a Raspberry Pi-based system that measures and accounts for ECG values as well as other patient health data, which could be valuable for future exams. 
P. Gupta, D. Aggarwal et al. [18] utilised an Intel Galeleo development board to collect data and upload it to a database where doctors may access it, reducing the need for patients to visit hospitals for routine examinations. K. Sahoo, S. K. Mohapatra, et al. [19] investigated and analysed a cloud-based health database in order to use threshold values to predict the possibility of a disease in a patient. P Kanade et al. [20], [21] have proposed remote monitoring intelligent robot device to maintain safe distance from affected patients without the risk of contamination.

\section{CONCLUSION}

We looked at the importance of the Internet of Things in healthcare and how it could help people manage with chronic illnesses and pandemics. From the frameworks and research, we can deduce that IoT can provide numerous benefits for smart hospitals, including low cost and high reliability, even in the event of pandemics, and that healthcare workers can take advantage of the majority of these benefits without coming into direct contact with infected people. As fresh facts surface on a regular basis, they continue to perceive a desire for low-cost management. We're getting close to bridging the gap between the actual, practical, and digital worlds. The Internet of Things is gradually delivering a wealth of modification applications in our daily lives, making life more easier for us. Fieldwork and case studies are conducted in order to achieve widespread acceptance of IoT without disclosing its formulation concerns, as well as to provide end users with secrecy and protection. The implementation of the Internet of Things demands strong and committed efforts. It purports to communicate even a minor adjustment in the user's life status. Thanks to a widely shared, localised complex scheme of intelligent gadgets, IoT has enabled considerable improvements to critical facilities in utilities, healthcare, education, and other areas, providing a modern scheme to technological advancement.

\section{REFERENCES}

[1] Wijethilaka, Shalitha, and Madhusanka Liyanage, "Survey on network slicing for internet of things realization in $5 \mathrm{~g}$ networks," IEEE Communications Surveys \& Tutorials, 2021.

[2] Gubbi, Jayavardhana, Rajkumar Buyya, Slaven Marusic, and Marimuthu Palaniswami, "Internet of Things (IoT): A vision, architectural elements, and future directions," Future generation computer systems, vol. 29, no. 7, pp. 1645-1660, 2013.

[3] Rahmani, Amir M., Tuan Nguyen Gia, Behailu Negash, Arman Anzanpour, Iman Azimi, Mingzhe Jiang, and Pasi Liljeberg, "Exploiting smart e-Health gateways at the edge of healthcare Internet-of-Things: A fog computing approach.," Future Generation Computer Systems, vol. 78, pp. 641-658, 2018.

[4] Xu, Boyi, Li Da Xu, Hongming Cai, Cheng Xie, Jingyuan Hu, and Fenglin $\mathrm{Bu}$, "Ubiquitous data accessing method in IoT-based information system for emergency medical services," IEEE Transactions on Industrial informatics, vol. 10, no. 2, pp. 1578-1586, 2014.

[5] Kumar, Harish, Manoj Kumar Singh, M. P. Gupta, and Jitendra Madaan, "Moving towards smart cities: Solutions that lead to the Smart City Transformation Framework," Technological forecasting and social change, vol. 153, p. 119281, 2020.

[6] Khan, Jamil Y., Dong Chen, and Oliver Hulin, "Enabling technologies for effective deployment of Internet of Things (IoT) systems: A communication networking perspective," Journal of Telecommunications and the Digital Economy, vol. 2, no. 4, pp. 65$1,2014$.

[7] Prakash Kanade, "Medical Assistant Robot ARM for Covid-19 Patients Treatment - A Raspberry Pi Project," International Research Journal of Engineering and Technology (IRJET), vol. 7, no. 10, 2020.

[8] Prakash Kanade, Prajna Alva, Jai Prakash Prasad, Sunay Kanade, "Smart Garbage Monitoring System using Internet of Things (IoT)," 2021 5th International Conference on Computing Methodologies and Communication (ICCMC), IEEE, pp. 330-335, 2021.

[9] Prakash Kanade, Sunay Kanade, "Medical Assistant Robot ARM for COVID-19 Patients Treatment - A Raspberry Pi Project," International Research Journal of Engineering and Technology (IRJET), vol. 7, no. 10, pp. 105-111, 2020.

[10] Prakash Kanade,Prajna Alva, "RASPBERRY PI PROJECT ULTRASONIC DISTANCE SENSOR IN CIVIL ENGINEERING," International Journal in IT \& Engineering (IJITE), vol. 8, no. 10, pp. 1-6, 2020.

[11] Prakash Kanade, Fortune David, Sunay Kanade, "Convolutional Neural Networks (CNN) based Eye-Gaze Tracking System using Machine Learning Algorithm," European Journal of Electrical Engineering and Computer Science, vol. 5, no. 2, pp. 36-40, 2021.

[12] Leng, Jichao, Zihuai Lin, and Peng Wang, "An implementation of an internet of things system for smart hospitals," in In 2020 IEEE/ACM Fifth International Conference on Internet-of-Things Design and Implementation (IoTDI), IEEE, pp. 254-255 2020.

[13] Fong, Ignatius W., ed, "Challenges in infectious diseases," Springer Science \& Business Media, 2012.

[14] Oshitani, Hitoshi, Taro Kamigaki, and Akira Suzuki, "Major issues and challenges of influenza pandemic preparedness in developing countries," Emerging infectious diseases, vol. 14, no. 6, p. 875, 2008.

[15] Ahn, Byeong-Gu, Yun-Hong Noh, and Do-Un Jeong, "Smart chair based on multi heart rate detection system," In 2015 IEEE SENSORS, pp. 1-4, 2015.

[16] Chiuchisan, Iuliana, Hariton-Nicolae Costin, and Oana Geman, "Adopting the internet of things technologies in health care systems," In 2014 International Conference and Exposition on Electrical and Power Engineering (EPE), pp. 532-535, 2014.

[17] Gupta, M. Surya Deekshith, Vamsikrishna Patchava, and Virginia Menezes, "Healthcare based on IoT using raspberry pi," in In 2015 International Conference on Green Computing and Internet of Things (ICGCIoT), IEEE, pp. 796-799, 2015.

[18] Gupta, Punit, Deepika Agrawal, Jasmeet Chhabra, and Pulkit Kumar Dhir, "Iot based smart healthcare kit," in In 2016 International Conference on Computational Techniques in Information and Communication Technologies (ICCTICT), IEEE, pp. 237-242, 2016.

[19] Sahoo, Prasan Kumar, Suvendu Kumar Mohapatra, and Shih-Lin $\mathrm{Wu}$, "Analyzing healthcare big data with prediction for future health condition,” IEEE Access, vol. 4, pp. 9786-9799, 2016.

[20] Prakash Kanade, Monis Akhtar, Fortune David, "Computer Networking and Technology Improvement in the Age of COVID-19,", International Journal of Advanced Networking and Applications (IJANA), vol. 12, no. 03, pp. 4592-4595, 2020.

[21] Prakash Kanade, Monis Akhtar, Fortune David, "Remote Monitoring Technology for COVID-19 Patients," European Journal of Electrical Engineering and Computer Science, vol. 5, no. 1, pp. 44-47, 2021. 